\title{
Case Report: Atypical Presentation and Intraoperative Complication in a Case of Aortic Dissection
}

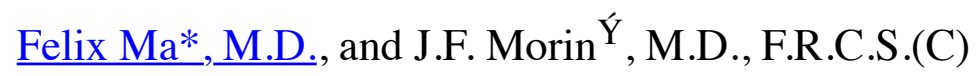

* To whom correspondence should be addressed: Department of Cardiovascular and Thoracic Surgery, Montreal General Hospital, McGill University, 1650 Cedar Av., Montreal, QC, Canada H3G 1A4

\section{THE CASE}

\section{Clinical Presentation}

A 68-year-old man, ex-smoker, with a history of hypertension, hypercholesterolemia, and intermittent claudication secondary to severe aorto-iliac occlusive disease, was evaluated by a cardiologist for exertional right-shoulder pain of one year's duration. The pain would typically last approximately five minutes and resolve with cessation of activity. He experienced no similar episodes at rest. There was no retrosternal chest pain, shortness of breath, orthopnea, paroxysmal nocturnal dyspnea, palpitations, transient ischemic attacks, syncope, or pre-syncope. His medications included Norvasc, Prinivil, Novoflupam, Nitrospray, Novo-Timol, Pravacho, Entrophen, and Tylenol. Family history was positive for coronary artery disease.

On physical examination, the patient was in no apparent distress with a blood pressure of 160/80 in both arms and a regular pulse of 70 . There was a left carotid bruit. Femoral pulses were nearly absent bilaterally. Heart sounds were normal, with no murmurs, and there were no signs of heart failure. EKG showed normal sinus rhythm and no evidence of ischemia or previous infarction. However, thallium scan revealed a fixed deficit in the inferior wall territory.

\section{Investigation}

Because the clinical picture suggested anginal equivalence, the patient was referred for cardiac catheterization. The angiogram revealed a normal left-ventricular ejection fraction, no mitral regurgitation, and a non-dominant right coronary artery with a $90 \%$ proximal lesion and a distal circumflex occlusion. Quite unexpectedly, during angiography, contrast dye was found to percolate into the anterior wall of the ascending aorta (Figure 1). This occured at two different sites, suggesting the presence of a communication between the lumen of the aorta and its wall. Thus, a type A aortic dissection had been discovered serendipitously.

Interestingly, a trans-esophageal echocardiogram (TEE) failed to confirm the finding. An area of ascending aortic wall thickening consistent with an atherosclerotic plaque had been reported, but no intimal flap was apparent. There was no evidence of aortic insufficiency or pericardial effusion. However, by contrast CT scan 
of the chest, the diagnosis of type A aortic dissection was confirmed. The CT demonstrated the presence of two distinct lumens with a visible intimal flap in the ascending aorta, possibly extending to the proximal aortic arch.

\section{Management}

Faced with an incidental finding of type A aortic dissection during a routine investigative coronary angiography in a patient with atypical clinical presentation, an aggressive approach for surgical intervention was adopted. Due to the presence of severe aorto-iliac occlusive disease, femoral arterial cannulation was not a viable option. Instead, right axillary artery and right atrial cannulation was selected for cardiopulmonary bypass. Upon inspection of the ascending aorta, there was no external evidence of dissection. The right axillary artery was exposed and cannulated in the usual fashion. Its wall appeared normal, with no evidence of hematoma or dissection.

However, after cross-clamping the aorta, difficulty was encountered in establishing proper arterial inflow via the right axillary artery. For reasons unknown at the time, the left subclavian artery and the proximal descending thoracic aorta were completely collapsed, with palpable pressure only in the innominate and left common carotid arteries. With more proximal repositioning of the cross-clamp, arterial inflow to the transverse arch and the proximal descending thoracic aorta was re-established. Apparently, the initial difficulty had been caused by entry of the cannula into the false lumen in the right subclavian artery. The aortic cross-clamp had excluded the entry point, thus confining blood flow and palpable pressures to the extent of the dissection in the innominate and left common carotid arteries.

Under profound hypothermic circulatory arrest, the ascending aorta was excised with its entry tear. The distal anastomosis was fashioned with a $28 \mathrm{~mm}$ Hemashield graft (open technique). Arrest time was 22 minutes. Antegrade flow was reestablished via the cross-clamped Hemashield graft, and proximal anastomosis was completed. The patient emerged from cardiopulmonary bypass without difficulties and with good hemodynamics.

The post-operative course was marked by transient atrial fibrillation, bilateral calf deep vein thrombosis, and right hemiparesis (on postoperative day 3). CT scan of the brain showed no evidence of intracranial bleeding or acute or subacute infarct. Carotid duplex scan revealed a prominent soft plaque in the left common carotid artery and bilateral internal carotid artery stenosis of up to $15 \%$. The patient was discharged two weeks after the operation. On follow-up two months later, he was able to walk without a cane.

\section{DISCUSSION}

Classically, a patient with acute aortic dissection presents with a history of sudden-onset excruciating, "tearing" anterior chest pain with or without radiation to the back. The pain is usually migratory, extending inferiorly along the length of the aorta to the abdomen or flank areas. In patients with chronic dissection, symptoms and signs are frequently related to the presence of an aortic aneurysm, particularly if the aneurysm is enlarging. If further dissection or aneurysm rupture occurs, the presentation may be similar to that of an acute dissection.

In this case, the patient presented with a one-year history of intermittent right shoulder pain with no other clinical features suggestive of either acute or chronic aortic dissection. The finding of aortic dissection was discovered incidentally upon cardiac catheterization for evaluation of coronary artery disease. Surprisingly, TEE, a very sensitive diagnostic modality, particularly for dissection of the ascending aorta (4), failed to confirm the diagnosis. However, CT scan of the chest was diagnostic. For timely diagnosis of a suspected aortic dissection, it is important to be familiar with the advantages and limitations of aortography, CT, MRI, and TEE in order to select the most appropriate imaging method. A review of diagnostic imaging modalities 
for the evaluation of suspected aortic dissection, comparing their relative sensitivities, specificities, and practicality, as well as the diagnostic information they provide, can be useful to the clinician (5).

Complications of a type A dissection may include sudden cardiovascular collapse from cardiac tamponade secondary to pericardial effusion, or massive acute myocardial infarction from dissection of coronary arteries, usually the right coronary artery. Aortic insufficiency may occur due to retrograde dissection into the aortic sinuses or annulus. A dissection may also be complicated by branch artery obstruction resulting in neurovascular deficits, such as stroke, ischemic neuropathy, paraplegia and paresis, limb ischemia, bowel ischemia, and renal failure.

In acute type A dissection, the high risk of mortality with medical treatment and the more favorable surgical outcomes mandate urgent surgical intervention (1-3). Surgery for chronic dissection is indicated for late aneurysm formation and chronic aortic insufficiency. Due to the unusual clinical presentation in this case, it is not clear whether the dissection was acute or chronic. Surgical intervention was chosen, although medical management with close follow-up using serial imaging studies might not have been unreasonable.

Successful preoperative selection of the site of arterial cannulation requires precise delineation of branch vessel involvement in the dissection. In this case, a proper preoperative angiogram of the aortic arch and its branches might have identified the false lumen in the right subclavian artery. With this key information, cannulation of the right subclavian artery might have been avoided, thereby eliminating the intraoperative complication and ensuring adequate cerebral and distal perfusion. In this case, the appropriate arterial cannulation site would have been the left axillary artery. Thus, this interesting case of aortic dissection not only demonstrates the possibility of atypical clinical presentation but also the importance of an appropriate preoperative angiogram in strategic planning for arterial cannulation.

\section{REFERENCES}

1. Cachera VP, Vouhe PR, Loisance DY, et al. Surgical management of acute dissections involving the ascending aorta: early and late results in 38 patients. Journal of Thoracic Cardiovascular Surgery 82: 576; 1981.

2. Miller DC. Surgical management of aortic dissections: indications, perioperative management and longterm results. In: Doroghazi RM, Slater EF, eds. Aortic Dissection. New York: McGraw-Hill; 1983.

3. Miller DC, Mitchell RS, Oyer PE, et al. Independent determinants of operative mortality for patients with aortic dissections. Circulation 70 (Suppl. 1): 1-153; 1984.

4. Nienaber CA, Von Kodolitsch Y, Nicolas V, et al. The diagnosis of thoracic aortic dissection by noninvasive imaging procedures. New England Journal of Medicine 328: 1-9; 1993.

5. Cigarroa JE, Isselbacher EM, DeSanctis RW, et al. Diagnostic imaging in the evaluation of suspected aortic dissection. New England Journal of Medicine 328: 35-43; 1993.

\section{BIOGRAPHY}

Felix Ma, M.D., received his M.D. at McGill University (Montreal, Quebec, Canada) in 1989 and completed his residency in General Surgery at McGill University. In June 1996 he will have completed his training in Cardiothoracic Surgery, and will pursue a clinical fellowship in Cardiac Surgery at the Cleveland Clinic (Cleveland, Ohio, USA). 
Copyright (C) 1996 by MJM 